\title{
DESARROLLO DE UN PROGRAMA PILOTO DE FARMACOVIGILANCIA EN EL HOSPITAL SAN JUAN DE DIOS DE ZIPAQUIRÁ
}

\section{DEVELOPMENT OF A PHARMACOVIGILANCE PILOT PROGRAM IN THE SAN JUAN DE DIOS HOSPITAL OF ZIPAQUIRA}

Gabriel Tribiño E. ${ }^{1}$

\section{RESUMEN}

Los programas hospitalarios de farmacovigilancia son indispensables para la consolidación de un programa nacional y para el mejoramiento continuo de la calidad de la atención hospitalaria. El presente estudio tuvo como objetivo desarrollar un programa piloto en un hospital de segundo nivel y caracterizar las reacciones adversas detectadas. El programa se presentó ante el Comité de Vigilancia Epidemiológica y los profesionales de los servicios incluidos (medicina interna, cirugía, ginecoobstetricia y urgencias). La información, se recolectó mediante notificación espontánea suministrada por profesionales del hospital y seguimiento intensivo por parte del investigador durante un período de seis meses (marzo-septiembre de 2006), empleando un formulario basado en el formato de reporte del INIVIMA. Se elaboraron reportes que se discutieron periódicamente en el comité. Se detectaron 60 reacciones adversas, de los cuales 48 ocurrieron en pacientes hospitalizados (incidencia acumulada 19x1000); ocho causaron la hospitalización del paciente y cuatro se detectaron en el servicio de urgencias. Las reacciones adversas a nivel gastrointestinal fueron las más reportadas en pacientes hospitalizados (27\%). La hipoglicemia fue la reacción adversa que con mayor frecuencia causó hospitalización

${ }^{1}$ MD, MSc. Farmacología, Especialista en Docencia Universitaria. Docente Facultad de Medicina Universidad de Ciencias Aplicadas y Ambientales U.D.C.A. Dirección para correspondencia: Calle 222 No. 55-37, Bogotá, D.C. gtrivino@udca.edu.co
(62,5\%). El $82 \%$ de los eventos adversos fueron reportados por el investigador y el $18 \%$ por personal del hospital. Algunas de las características clínicas de las reacciones adversas detectadas son similares a las reportadas en estudios realizados en instituciones de tercer nivel. El índice de notificación espontánea fue bajo. Las características del programa, se adaptan a las condiciones de una institución de segundo nivel y permiten su continuidad.

Palabras clave: Farmacoepidemiología, efectos adversos, vigilancia epidemiológica, proyectos piloto, hospitales públicos.

\section{SUMMARY}

Pharmacovigilance hospital programs play a key role in the consolidation of a national program and the continuous improvement of health care. The goal of this study was to develop a pharmacovigilance pilot program in a level two hospital and identify the salient clinical features of the adverse reactions detected. The program was presented before the hospital's Epidemiologic Surveillance Committee and the professionals of the wards included (internal medicine, surgery, obstetrics and gynecology and emergency). Information was collected by spontaneous reporting by the medical staff and intensive follow-up, carried out by the researcher during a six month period (march-september 2006). A format based on the INVIMA reporting sheet was used. Reports presented periodically to the committee were produced. Sixty adverse drug reactions were detected, of which 48 occurred in hospitalized patients 
(incidence 19X1000); eight led to hospitalization and four were detected in the emergency ward. Adverse reactions affecting gastrointestinal system were the most frequent ones reported in hospitalized patients (27\%). Hypoglycemia was the adverse reaction that more often led to hospitalization (62.5\%). $82 \%$ of the adverse reactions were reported by the author and $18 \%$ by the medical staff. Some of the clinical features of the adverse reactions detected are similar to those found in studies performed at level three hospitals. The rate of spontaneous notification was low. The features of the program match those of a second level hospital and allow its continuity.

Key words: Pharmacoepidemiology, adverse effects, epidemiologic surveillance, pilot projects, hospitals, public.

\section{INTRODUCCIÓN}

Los medicamentos han sido factor clave en la erradicación o el control de ciertas enfermedades, en el bienestar general de la población y en el incremento en la esperanza de vida (Segura E Maldonado, 2003). A pesar de estos beneficios, no es posible desconocer que ellos producen efectos adversos, incluso, cuando se usan correctamente. Desde el Proyecto Colaborativo de Vigilancia de Medicamentos de Boston, a comienzos de la década de 1970, las reacciones adversas a medicamentos (RAMs), se han reconocido como un problema clínico frecuente y una causa importante de morbilidad (Pirmohamed et al. 1999). Diversas publicaciones han demostrado que las RAMs afectan una proporción importante de pacientes hospitalizados y son responsables de un número considerable de hospitalizaciones y muertes anualmente (Johnson E Bootman, 1995; Lazarou et al. 1998; Ajayi et al. 2000). Además, incrementan en forma considerable los costos de la atención en salud (Segura $E$ Maldonado, 2003). Lo anterior evidencia la necesidad de establecer programas operativos de farmacovigilancia vinculados con el quehacer cotidiano de las instituciones de salud. De hecho, la resolución 1403 de 2007 del Ministerio de Protección Social señala que "las Instituciones Prestadoras de Servicios de Salud y las Empresas Administradoras de Planes de Beneficios, deberán contar con un programa institucional de Famacovigilancia, con una perspectiva especialmente clínica/individual que permita establecer y prevenir problemas relacionados con la indicación, efectividad y seguridad de los medicamentos". En Colombia, el desarrollo de tales programas, se ha dificultado por el desconocimiento de los objetivos, métodos y alcances de la farmacovigilancia y por la falta de interés de las instituciones y profesionales de la salud.

El objetivo de este estudio fue desarrollar un programa piloto de farmacovigilancia en una institución de segundo nivel, con características que permitieran el establecimiento de un programa definitivo, identificando, además, las principales características clínicas de las reacciones adversas detectadas.

\section{MATERIALES Y MÉTODOS}

Población de estudio: El estudio, se llevó a cabo en el Hospital San Juan de Dios de Zipaquirá (Cundinamarca, Colombia), un hospital del Estado que cuenta con 116 camas. Se incluyeron los pacientes hospitalizados en los servicios de medicina interna, cirugía y ginecoobstetricia, así como los atendidos en el servicio de urgencias (pacientes atendidos en consulta y en sala de observación), durante un periodo de seis meses (marzo primero a septiembre primero de 2006). También se incluyeron pacientes hospitalizados por causa de una RAM. Durante el período de estudio, se hospitalizaron 2.504 pacientes en los servicios mencionados, mientras que se atendieron 13.187 pacientes en el servicio de urgencias.

Presentación y sensibilización: El proyecto fue revisado por el Comité de Vigilancia Epidemiológica (COVE); cabe anotar que la institución donde se desarrolló el estudio no cuenta con comité de farmacia y terapéutica ni con comité de ética. Posteriormente, se realizó una presentación ante los integrantes del comité para precisar algunos aspectos referentes a la operatividad del programa y al formato de reporte de eventos adversos: se acordó que los formatos de reporte quedarían en las estaciones de enfermería de los servicios participantes; la enfermera del comité sería la encargada de la provisión de los formatos de reporte y el acopio de los ya diligenciados, para lo cual, se desplazaría a los diferentes servicios tres veces por semana; bimensualmente, se presentaría ante el COVE un consolidado de los datos obtenidos para efectuar el análisis correspondiente, evaluar la operatividad del programa y tomar las medidas pertinentes; se podrían realizar reuniones extraordinarias si la situación lo ameritaba (RAMs serias o frecuentes); se 
realizaron algunas modificaciones al formato de reporte original, para facilitar su diligenciamiento por parte del personal del hospital y, por último, se acordó que el investigador elaboraría al tercer y al sexto mes, con fines de retroalimentación, dos boletines informativos sobre las RAMs detectadas y la operatividad del programa, dirigidos a los profesionales del hospital y se presentarían durante la entrega general de turno y se fijarían en las carteleras de la institución.

Luego de esta presentación ante el COVE, se realizó una sesión de sensibilización, en la cual, se contó con la presencia de los médicos de planta, los médicos internos y las enfermeras de los servicios participantes. Los objetivos de esta sesión fueron resaltar la importancia de la farmacovigilancia, precisar los conceptos de evento adverso y reacción adversa a medicamento y explicar el funcionamiento general del programa piloto haciendo énfasis en la importancia de la participación de los profesionales de la salud y la manera de diligenciar el formato de reporte.

Definición de caso: Para detectar las reacciones adversas, se empleó una estrategia pasiva y una activa. La pasiva, se basó en el reporte espontáneo de los eventos adversos por parte de los profesionales de la salud (médicos, internos, enfermeras), mediante el diligenciamiento del formato de reporte, que eran recogidos por la enfermera del COVE y entregados luego al investigador, quien evaluaba su calidad y completaba la información, si era indispensable. La estrategia activa estuvo a cargo del investigador, quien se desplazó dos veces por semana a los servicios incluidos en el estudio, para revisar las historias clínicas en busca de signos y/o síntomas que apuntaran hacia la presentación de un evento adverso, realizando entrevistas o examen físico en caso de ser necesario. Los eventos detectados mediante esta estrategia, se confirmaron con los médicos tratantes. La enfermera del COVE y el investigador tenían como función adicional, estimular la notificación espontánea durante sus visitas a los diferentes servicios.

Se acogió la definición de reacciones adversas a medicamentos establecida por el reporte técnico 498 de la OMS (The Uppsala Monitoring Centre). Las reacciones adversas a medicamentos, se caracterizaron con la terminología de reacciones adversas de la OMS (WHOART, The Uppsala Monitoring Centre, 2006). Después de identificar los eventos adversos, se aplicó el algoritmo de Naranjo, para generar la probabilidad de causalidad (Naranjo \& Busto, 1992). Las reacciones adversas a medicamentos clasificadas como definitivas, posibles o probables, se tuvieron en cuenta para el análisis; las clasificadas como dudosas fueron excluidas.

Análisis de datos: Los datos recopilados, se analizaron para estudiar las siguientes características de las reacciones adversas: medicamentos implicados, manifestaciones clínicas, órganos / sistemas afectados, tipo, causalidad y gravedad de la reacción adversa, cambios en los medicamentos implicados, desenlace y diagnóstico principal del caso. Los medicamentos implicados, se definieron según la clasificación ATC (WHO Collaborating Centre for Drug Statistics Methodology). El tipo de reacciones adversas a medicamentos, se determinó según la clasificación de Rawlins y Thompson: A, augmented (incrementada); B, bizarre (rara); E, end (terminal) (Bustamante, 2001). El diagnóstico principal de los casos, se definió según la CIE-10. La gravedad, se clasificó según las definiciones de Naranjo (Naranjo E Busto, 1992).

Los datos necesarios para el cálculo de incidencias y proporciones fueron suministrados por el departamento de estadística del hospital. La base de datos y el análisis, se realizó en Excel 2003.

\section{RESULTADOS Y DISCUSIÓN}

La presente investigación constituye uno de los primeros estudios de farmacovigilancia llevados a cabo en el país, en una institución de segundo nivel. En la bibliográfica consultada, se evidenció que en Colombia la mayoría de estos estudios se han efectuado en el tercer nivel de atención; solamente se encontró una investigación llevada a cabo en un hospital de segundo nivel de Bogotá, la cual analizó RAMs ocurridas en pacientes que asistieron a consulta externa y al servicio de urgencias (Moscoso et al. 2006). Es necesario tener en cuenta lo anterior en el momento de efectuar comparaciones entre los resultados de las diferentes investigaciones.

Frecuencia: Durante los seis meses del programa piloto, se detectaron un total de 60 RAMs, en 55 pacientes, discriminadas de la siguiente forma: 48 RAMs, el $80 \%$ del total, ocurrieron en 44 pacientes hospitalizados; ocho RAMs, $13,3 \%$, causaron la hospitalización del paciente y cuatro, $6,7 \%$, se manifestaron en el servicio de 
urgencias. Presentaron una RAM 51 de los 55 pacientes, correspondiente al 92,7\%; tres pacientes, 5,4\%, presentaron dos reacciones adversas y un paciente, $1,8 \%$, presentó tres reacciones adversas. Un paciente que se hospitalizó a causa de una RAM presentó otra reacción adversa durante su estancia hospitalaria.

Respecto a la frecuencia de reacciones adversas por servicio, la mayor proporción de casos se detectó en medicina interna (86\%), seguido por urgencias $(7 \%)$, cirugía (5\%) y ginecoobstetricia (2\%). La incidencia de RAMs en medicina interna fue 5,8\%, en cirugía $0,7 \%$ y en ginecoobstetricia $0,14 \%$.

Teniendo en cuenta el total de egresos de los servicios incluidos durante los seis meses de duración del estudio piloto (sin considerar urgencias), la incidencia acumulada de RAMs en pacientes hospitalizados fue de 19 X 1000 (48/2504).

Considerando que se presentaron ocho ingresos a medicina interna a causa de una reacción adversa, éstas dieron cuenta del $0,9 \%$ de las admisiones a este servicio.

La incidencia de RAMs en pacientes hospitalizados encontrada en este estudio (1,9\%), se ubica dentro del rango reportado en la literatura (1,5\% - 35\%) (Karch \& Lasagna, 1975; Lazarou et al. 1998) y es cercana a la hallada por Classen et al. (1997), 2,4\%, pero lejana de la reportada por Pirmohamed et al. (1999), 10\% - 20\%. Cabe anotar que la incidencia calculada corresponde a los servicios de medicina interna, cirugía y ginecoobstetricia, por lo cual, no es la incidencia global para el hospital, ya que no se incluyó el servicio de pediatría. Por otra parte, considerando que uno de los problemas de los sistemas de notificación espontánea es el bajo índice de reportes es probable que la incidencia real sea mayor que la encontrada.

La incidencia de RAMs en pacientes hospitalizados en medicina interna, sin contar los ingresos causados por RAMs, fue más baja que la reportada por otros estudios realizados en servicios de medicina interna de instituciones de tercer nivel: en un estudio realizado en el Hospital de Caldas (Miranda et al. 2003), se reportó una incidencia de $7 \%$, mientras que en un estudio realizado en un hospital de tercer nivel de Bogotá (Tribiño et al. 2006), se encontró un $25 \%$. Las diferencias en las incidencias reportadas por diferentes publicaciones reflejan diferencias en las poblaciones de pacientes, criterios para evaluar reacciones adversas y técnicas de vigilancia. En los servicios de cirugía y ginecoobstetricia, la incidencia fue muy baja, lo cual, es compatible con lo reportado en la literatura.

\section{RAMs en pacientes hospitalizados}

Características generales. La media de la edad de los pacientes que presentaron RAMs durante su hospitalización fue 65,9 $\pm 16,6$ años. La mayor proporción de RAMs, $34 \%$, ocurrió en pacientes entre los 70 y los 80 años. El $73 \%$ de estos pacientes (32) eran de sexo femenino. A este respecto vale la pena anotar que durante el período de estudio, en los servicios incluidos excepto urgencias, se atendió una mayor cantidad de mujeres que de hombres. Al comparar las proporciones de hombres y mujeres con RAMs, no se encontró una diferencia estadísticamente significativa $(p=0,22)$.

La gran mayoría de las reacciones adversas detectadas fueron de tipo A (89,6\%); el 8,4\% del tipo B y solamente el $2 \%$ fue de tipo E. La mayor proporción de reacciones adversas, se clasificó como probable $(89,6 \%$ y el restante $10,4 \%$ como posible. En cuanto a la severidad, 46 de las 48 reacciones adversas (95\%), se clasificaron como moderadas; los otros dos casos correspondieron a una reacción leve (hipokalemia relacionada con ampicilina sulbactam) y una letal (shock anafiláctico causado por dipirona). Respecto al desenlace, la mayoría de las reacciones adversas (91,7\%), se resolvieron sin dejar secuelas; en el 6,3\% de los casos, la manifestación persistía al egreso del paciente y una, el $2 \%$, causó la muerte del paciente.

Los hallazgos anotados respecto a tipo, causalidad y severidad son compatibles con lo reportado en la literatura (Segura \& Maldonado, 2003; Classen et al. 1997; Suh et al. 2000; Gholami E Shalviri 1999). El predominio de RAMs tipo A indica que la mayoría son predecibles. El carácter moderado de la mayoría de las reacciones adversas coincide con la recuperación sin secuelas de la mayoría, hallazgo similar al del estudio de Churl et al. (2000), el cual, encontró recuperación completa en el $80 \%$ de las RAMs detectadas.

Manifestaciones clínicas. Las reacciones adversas a medicamentos comprendieron 20 manifestaciones diferentes; las más frecuentes se presentan en la tabla 1. Los sistemas afectados con mayor frecuencia fueron: gastrointestinal (27\%), renal $(22,9 \%)$, endocrino $(16,6 \%)$ y hematológico $(14,5 \%)$. 
Tabla 1. Reacciones adversas en pacientes hospitalizados.

\begin{tabular}{|c|c|c|c|}
\hline RAM & Frecuencia & $\%$ & Medicamentos implicados \\
\hline Hipopotasemia & 9 & 18,7 & $\begin{array}{c}\text { Furosemida (4), hidrocortisona (3), hidroclorotiazida } \\
\text { (1),ampicilina sulbactam (1) }\end{array}$ \\
\hline Sobreanticoagulación & 7 & 14,5 & Warfarina (4), heparina no fraccionada (3) \\
\hline Hipoglicemia & 6 & 12,5 & Insulina (5), glibenclamida (1) \\
\hline Emesis & 4 & 8,3 & Tramadol (3), dicloxacilina (1) \\
\hline Hiperkalemia & 3 & 6,2 & Espironolactona (3) \\
\hline Abdomen, dolor zona superior & 3 & 6,2 & Digoxina (2), eritromicina (1) \\
\hline Erupción cutánea & 2 & 4,1 & Ampicilina (1), oxacilina (1) \\
\hline Dispepsia & 2 & 4,1 & Aspirina (1), teofilina (1) \\
\hline Otras & 12 & 25 & \\
\hline TOTAL & 48 & 100 & \\
\hline
\end{tabular}

En varios estudios en pacientes hospitalizados (Bates et al. 1997; Dennis et al. 1998; Classen et al. 1991), las manifestaciones cutáneas fueron las más frecuentes, con porcentajes entre 24 y $28 \%$; esto contrasta con los hallazgos de esta investigación, en la cual, dichas manifestaciones se presentaron solo en el $2 \%$ de los casos. En estudios realizados en el país los resultados son variables. Los que se llevaron a cabo en el Hospital de Caldas (Miranda et al. 2003) y en el Hospital de San Ignacio (Dennis et al. 1998), las reacciones adversas cutáneas se presentaron con una frecuencia importante, mayor que la hallada en el presente estudio: $28 \%$ en la primera institución, siendo. en este caso, la manifestación más frecuente y $20,7 \%$ en la otra, que para esta institución fue la segunda más frecuente. En contraste, en la investigación realizada en un hospital de tercer nivel de Bogotá (Tribiño et al. 2006) las RAMs cutáneas representaron apenas un 2,2\% de los casos.

La frecuencia de hipopotasemia en estudios internacionales es menor que la detectada en esta investigación. Llama la atención que en estudios realizados en Colombia esta manifestación figura entre las reacciones adversas más reportadas: en el estudio del Hospital de Caldas la hipopotasemia inducida por diuréticos fue el evento adverso más frecuente ( $44,8 \%$ de los casos), mientras que en el estudio del hospital de tercer nivel de Bogotá fue el segundo (19,4\%) (Miranda et al. 2003). La hipoglicemia, se reporta con baja frecuencia en publicaciones internacionales; sin embargo, en el estudio del hospital de tercer nivel de Bogotá constituyó la tercera RAM más frecuente, con un 7\% de los casos (Tribiño et al. 2006).

Las tres reacciones adversas detectadas con mayor frecuencia en este estudio son totalmente predecibles y pueden ser, por lo menos, en cierta proporción, prevenibles. Vale la pena que el servicio de medicina interna del hospital realice una revisión de historias clínicas y discusión de las mismas con los médicos del servicio, que bien se podría llevar al comité de vigilancia epidemiológica; lo anterior, con el fin de evaluar la existencia de causas prevenibles de estas RAMs (dosificación sin tener en cuenta el peso del paciente, en el caso de heparina no fraccionada e insulina, control paraclínico inadecuado, ajuste de dosis inadecuado, según la evolución) y tomar las medidas pertinentes para evitarlas.

Otros ejemplos de reacciones adversas potencialmente prevenibles fueron: emesis por tramadol endovenoso, hiperpotasemia por espironolactona, empeoramiento de broncoespasmo por betabloqueador en paciente con EPOC, epistaxis por warfarina, crisis de gota relacionada con furosemida en paciente con artritis gotosa e hipertensión de rebote por suspensión abrupta de clonidina. 
Medicamentos implicados. 23 diferentes medicamentos generaron reacciones adversas en pacientes hospitalizados. Los más frecuentemente implicados fueron: furosemida e insulina, relacionados cada uno con el $10,4 \%$ de los casos; hidrocortisona, tramadol y warfarina vinculados cada uno con el 8,3\% de los casos; digoxina, espironolactona y heparina no fraccionada asociados cada uno con el 6,3\% de las reacciones adversas. Los grupos anatomoterapéuticos más implicados fueron: medicamentos que actúan en el sistema cardiovascular, los cuales, causaron el $31,2 \%$ de los casos; medicamentos que actúan en aparato digestivo y metabolismo, medicamentos que actúan en sangre y antiinfecciosos, los cuales, causaron cada uno el $14,6 \%$ de las reacciones adversas $\mathrm{y}$, finalmente, medicamentos que actúan en el sistema nervioso central, asociados con el $12,5 \%$ de los casos. Cabe destacar que la mayoría de RAMs relacionadas con medicamentos que actúan en el sistema cardiovascular fueron causadas por diuréticos (60\%). Casi todas las RAMs asociadas con medicamentos del grupo de aparato digestivo y metabolismo fueron generadas por insulina y sulfonilureas (86\%). Todas las RAMs relacionadas con medicamentos que actúan en sangre fueron causadas por warfarina y heparina no fraccionada. Finalmente, el 57,1\% de las reacciones adversas asociadas con antiinfecciosos fue causado por betalactámicos.

A diferencia de trabajos donde los anti-infecciosos son los agentes más implicados en el desarrollo de RAMs (Churl et al. 2000; Dennis et al. 1998; Lagnaoui et al. 2000), en esta investigación los medicamentos con acción en el sistema cardiovascular fueron los más frecuentemente relacionados. En otros estudios, los agentes cardiovasculares se han relacionado con 17 $36 \%$ de las RAMs detectadas (Gholami et al. 1999; Churl et al. 2000), rango en el que está la proporción hallada por el presente estudio.

Los anticoagulantes, se reportan también en diversas publicaciones con una frecuencia algo menor que en esta investigación (9,3\% - 13,8\%), Miranda et al. 2003; Bordet et al. 2001; Classen et al. 1991). Sin embargo, en el estudio realizado en un hospital de tercer nivel de Bogotá, se encontró que estos medicamentos fueron los principales causantes de RAMs, dando cuenta del 36,5\% de los casos detectados (Tribiño et al. 2006).

En general, los medicamentos asociados con mayor frecuencia a reacciones adversas en los diferentes estudios varían según la composición de la población estudiada y la frecuencia con la cual se prescriben las diversas clases de medicamentos, en esa población particular. Sin embargo, los grupos de medicamentos que más se conservan de un estudio a otro son anti infecciosos, cardiovasculares, anticoagulantes y analgésicos.

RAMs que generaron hospitalización: En cuanto a los pacientes afectados por estas RAMs, cinco $(62,5 \%)$ eran de sexo masculino y seis pacientes (75\%) tenían edades entre los 70 y los 82 años, siendo el promedio de edad 65,2 $\pm 20,3$ años. Las manifestaciones clínicas y los medicamentos implicados, se presentan en la tabla 2. Al aplicar el algoritmo de Naranjo, siete de las ocho reacciones adversas, se catalogaron como probables. Un caso de hipoglicemia por insulina fue clasificado como definitivo, ya que la manifestación recurrió tras la reexposición al fármaco implicado. En cuanto a la severidad, una de estas RAMs, se consideró grave (hipoglicemia severa con compromiso del sensorio relacionada con insulina), mientras que las demás, se catalogaron como moderadas, puesto que no amenazaron la vida ni dejaron secuelas. La única reacción adversa tipo $B$ fue el síndrome de hipersensibilidad a anticonvulsivantes; las demás fueron tipo A.

La proporción de admisiones por RAMs en la población estudiada fue $0,31 \%$, aunque esta cifra en realidad podría ser mayor, teniendo en cuenta que no se incluyó el servicio de pediatría y las limitaciones comentadas en el sistema de notificación espontánea.

Diversos estudios reportan que las reacciones adversas que con mayor frecuencia generan hospitalización son eventos hemorrágicos, arritmias, hipotensión arterial, hipoglicemia, insuficiencia renal y trastornos electrolíticos (Pirmohamed et al. 2004; Van der Hooft et al. 2008). Una revisión sistemática de estudios prospectivos (Kongkaew et al. 2008) mostró que la proporción de admisiones hospitalarias por reacciones adversas oscila entre $0,13 \%$ y $15,7 \%$, con una mediana de 5,3\%; los autores concluyen, además, que dicha proporción varía entre grupos etáreos, siendo mayor en ancianos. La casuística encontrada es limitada para hacer comparaciones, pero se observan algunas tendencias similares en la actual población estudiada.

RAMs en el servicio de urgencias: Las RAMs detectadas en este servicio fueron: dos casos de erupción 
Tabla 2. RAMs que generaron hospitalización.

\begin{tabular}{|l|c|c|c|}
\hline \multicolumn{1}{|c|}{ Reacción adverse } & Frecuencia & $\%$ & Medicamento implicado \\
\hline Hipoglicemia & 5 & 62,5 & $\begin{array}{c}\text { Glibenclamida (3), } \\
\text { insulina (2) }\end{array}$ \\
\hline Epistaxis & 1 & 12,5 & Warfarina \\
\hline $\begin{array}{l}\text { Síndrome de hipersensibilidad a los } \\
\text { anticonvulsivantes }\end{array}$ & 1 & 12,5 & Carbamazepina \\
\hline Sobreanticoagulación & 1 & 12,5 & \\
\hline \multicolumn{1}{|c|}{ TOTAL } & $\mathbf{8}$ & 100 & \\
\hline
\end{tabular}

eritematosa asociados con diclofenac y amoxicilina; un caso de hipotensión arterial relacionado con nitroprusiato de sodio y un caso de taquicardia supraventricular asociado a pseudoefedrina. La hipotensión asociada a nitroprusiato de sodio, se presentó en un paciente que era atendido en observación; las otras reacciones adversas originaron la consulta al servicio. En cuanto a la causalidad, la taquicardia supraventricular se clasificó como posible, mientras las demás reacciones adversas, se catalogaron como probables. En cuanto a la severidad, todas se consideraron moderadas. La hipotensión y la taquicardia supraventricular se resolvieron sin secuelas; los pacientes que presentaron exantemas egresaron luego de la consulta.

Las reacciones adversas en los servicios de urgencias han sido menos estudiadas (Hafner et al. 2002) y sus características varían según el tipo de hospital. Se reporta que las RAMs dan cuenta del $1,7 \%$ al $4,1 \%$ de las consultas a los servicios de urgencias, siendo las manifestaciones más frecuentes trastornos gastrointestinales y reacciones alérgicas relacionadas con diferentes grupos farmacológicos, así como eventos hemorrágicos e hipoglicemia asociados a anticoagulantes y antidiabéticos, respectivamente (Hafner et al. 2002; Yee et al. 2005). Los pocos casos detectados en esta investigación no permiten realizar comparaciones.

Operatividad del programa piloto: En este aspecto, se detectaron los siguientes problemas:

- En las presentaciones realizadas ante el COVE no siempre fue posible contar con la presencia de un médico representante de los servicios incluidos en el programa.

- Por tratarse de un programa piloto, el COVE consideró conveniente no reportar las RAMs a las entidades reguladoras; Sin embargo, para el funcionamiento adecuado de un programa establecido, es indispensable el reporte a las entidades pertinentes.

- A pesar de haber realizado una sensibilización antes del inicio del programa, el índice de notificación espontánea fue bajo. La mayor proporción de casos fue reportada por el investigador (82\%); los médicos de planta y los médicos internos reportaron, cada uno, un $8 \%$ de los casos y las enfermeras; el $2 \%$. En total, el personal del hospital realizó once reportes durante el programa piloto. Llama la atención que esta cifra coincide con el total de reportes captados por el programa piloto desarrollado en el Hospital San Ignacio de Bogotá (Dennis et al. 1998).

Un factor que pudo incidir en el bajo número de notificaciones es la escasa formación de los profesionales en farmacovigilancia. Aunque no se empleó un instrumento para medir los conocimientos del personal de la institución en esta disciplina, por no ser el objetivo del estudio, durante la interacción con ellos fue posible percibir este problema. Por ejemplo, buena parte de ellos, especialmente enfermeras y médicos internos, asocian reacción adversa únicamente con manifestaciones de hipersensibilidad. Adicionalmente, el programa piloto no contempló, adecuadamente, la sensibilización al personal nuevo (especialmente, médicos internos y 
rurales), lo cual, también pudo influir de forma negativa en el número de reportes captados.

En cuanto a las fortalezas del programa cabe destacar:

- El programa se adapta a las características funcionales de la institución, lo que favorece su continuidad.

- El programa resulta costo-efectivo, porque su implementación no exige la inversión de grandes recursos, mientras que su operatividad permitiría la detección temprana de eventos adversos, la detección de reacciones adversas prevenibles y el planteamiento de medidas para evitarlas.

- Luego de la presentación del informe final ante el COVE, se asignaron funciones de farmacovigilancia a algunos de los integrantes del comité y se acordó iniciar el reporte de las reacciones adversas detectadas al INVIMA. Esto también favorece la continuidad del programa.

- El personal sensibilizado favorece la continuidad del programa, lo cual se evidenció por la recepción de varios reportes luego de la finalización del programa piloto.

\section{CONCLUSIONES}

Algunas características de las RAMs detectadas (tipo, causalidad, severidad, desenlace) son similares a las reportadas en estudios internacionales y realizados en instituciones de tercer nivel de nuestro país, aunque otras (incidencia, medicamentos implicados, manifestaciones clínicas), difieren de lo reportado en la literatura. Esta variabilidad obedece a diversos factores, como población incluida y métodos de detección y evaluación de reacciones adversas.

El programa piloto desarrollado siguió los lineamientos de la OMS para llevar a cabo actividades de farmacovigilancia, se adaptó a las características de un hospital de segundo nivel, fue compatible con las actividades del COVE, despertó el interés de la institución por establecer un programa en forma definitiva y contribuyó a fomentar la cultura del reporte, lo cual, se evidenció por la recepción de varias notificaciones, luego de finalizar el programa. Sin embargo, para garantizar su continuidad y su operatividad es necesario efectuar algunos ajustes. Se deben implementar estrategias para asegurar la sensibilización de todos los profesionales de salud de la institución, incluyendo el personal nuevo. Es indispensable vincular de manera más estrecha a los especialistas, no sólo por medio del reporte espontáneo, sino también mediante su participación activa en las discusiones sobre farmacovigilancia que se lleven a cabo al interior del COVE. Adicionalmente, es convenciente que los integrantes del comité con funciones de farmacovigilancia continúen estimulando la notificación espontánea y mantengan la retroalimentación a los profesionales de los diferentes servicios.

Por otra parte es importante incluir los principios básicos de la farmacovigilancia en la cátedra de farmacología para medicina y enfermería, con el objeto de lograr un adecuado desempeño de los egresados dentro de los programas institucionales.

\section{BIBLIOGRAFÍA}

AJAYI, F.; SUN, H.; PERRY, J. 2000. Adverse drug reactions: a review of relevant factors. J. Clin. Pharmacol. 40:1093-1101.

BATES, D.; SPELL, N.; CULLEN, D.; BURDICK, E; LAIRD, N.; PETERSEN, L; SMALL, S.; SWEITZER, B.; LEAPE, L. 1997. The costs of adverse drug reactions in hospitalized patients. JAMA. 277:307-311

BORDET, R.; GAUTIER, S.; LE LOUET, H.; DUPUIS, B.; CARON, J. 2001. Analysis of the direct cost of adverse drug reactions in hospitalized patients. Eur. J. Clin. Pharmacol. 56:935-941.

BUSTAMANTE, C. 2001. Fases del desarrollo de un nuevo fármaco. En: Ardila, E.; Sánchez, R.; Echeverry, J. (eds). Estrategias de investigación en medicina clínica. Bogotá. Manual Moderno. p.131.

CHURL, D.; WOODALL, B.; SHIN, S.; HERMES DE SANTIS, E. 2000. Clinical and economic impact of adverse drug reactions in hospitalized patients. Ann. Pharmacoter. 34:1373-1379

CLASSEN, D.; PESTOTNIK, S.; EVANS, R.; BURKE, J. 1991. Computerized surveillance of adverse drug events in hospital patients. JAMA. 266(20):28472851. 
CLASSEN, D.; PESTOTNIK, S.; SCOTT EVANS, R.; LLOYD, J.; BURKE, J. 1997. Adverse drug events in hospitalized patients: excess length of stay, extra costs and attributable mortality. JAMA. 277(4):301-305.

DENNIS, R.; GUTIÉRREZ, J.; RODRÍGUEZ, M. 1998. Creación de un programa piloto de farmacovigilancia en el Hospital Universitario San Ignacio. Acta Médica Colombiana. 23(1):15-22.

GHOLAMI. K.; SHALVIRI, G. 1999. Factors associated with preventability, predictability and severity of adverse drug reactions. Ann. Pharmacother. 33:236-240.

HAFNER, J.; BELKNAP, S.; SQUILLANTE, M.; BUCHEIT, K. 2002. Adverse drug events in emergency department patients. Ann Emerg Med. 39(3):258-267.

JOHNSON, J.; BOOTMAN, J. 1995. Drug-related morbidity and mortality: a cost-of-illness model. Arch. Intern. Med. 155:1949-1956.

KARCH, F.E.; LASAGNA, L. 1975. Adverse Drug Reactions. JAMA. 234:1236-1241.

KONGKAEW, C.; NOYCE, P.; ASHCROFT, D. 2008. Hospital admissions associated with adverse drug reactions: a systematic review of prospective observational studies. Ann Pharmacother. 42(7):1017-1025.

LAGNAOUI, R.; MOORE, N.; FACH, J.; LONGYBOUSIER, M.; BÉGAUD, B. 2000. Adverse drug reactions in a department of systemic disease oriented intern medicine: prevalence, incidence, costs and avoidability. Eur. J. Clin. Pharmacol. 56(2):181-186.

LAZAROU, J.; POMERANZ, B.; COREY, P. 1998. Incidence of adverse drug reactions: a meta-analysis of prospective studies. JAMA. 279:1200-1205.

MINISTERIO DE LA PROTECCION SOCIAL. Resolución 1403 de 2007. Disponible desde Internet en: http://www.minproteccionsocial.gov.co/VBeContent/library/documents/DocNewsNo17379Docu mentNo6819.PDF. (con acceso 09/06/08).
MIRANDA, H.; BURITICA, O.; AYALA, O. 2003. Eventos adversos a medicamentos en los servicios de medicina interna del Hospital de Caldas. Rev. Médica de Risaralda. 9(1):1-7.

MOSCOSO, S.; RAMIREZ, G.; LÓPEZ, J.; GERENA, B. 2006. Reacciones adversas a medicamentos en el Hospital de Suba de Bogotá. Rev. Salud Pública. 8 (2):209-217.

NARANJO, C.; BUSTO, U. 1992. Reacciones adversas a medicamentos. En: Métodos en farmacología clínica. Santiago: OPS. p.331.

PIRMOHAMED, M.; BRECKENRIDGE, A.; KITTERINGHAM, N. 1999. Adverse drug reactions. BMJ. 316:1295-1298.

PIRMOHAMED, M.; JAMES, S.; MEAKIN, S.; GREEN, C.; SCOTT, A.; WALLEY, T.; FARRAR, K.; PARK, B.; BRECKENRIDGE, A. 2004. Adverse drug reactions as cause of admission to hospital: prospective analysis of 18820 patients. BMJ. 329(7456):15-19.

SEGURA, O.; MALDONADO, C. 2003. Las reacciones adversas a medicamentos: una aproximación desde el punto de vista económico. Biomédica. 23:401-407.

SUH, D.C.; WOODALL, B.; SHIN, S.K.; HERMES-DE SANTIS, E.R. 2000. Clinical and economic impact of adverse drug reactions in hospitalized patients. Ann. Pharmacoter. 34:1373-1379.

THE UPPSALA MONITORING CENTRE. Glossary of terms used in pharmacovigilance. Disponible desde Internet en: http://www.who-umc.org/graphics/15338.pdf. (con acceso 03/03/06).

TRIBIÑO, G.; MALDONADO, C.; SEGURA, O.; DIAZ, J. 2006. Costos directos y aspectos clínicos de las reacciones adversas a medicamentos en pacientes hospitalizados en el servicio de medicina interna de una institución de tercer nivel de la ciudad de Bogotá. Biomédica. 26:31-41.

VAN DER HOOFT, C; DIELEMAN, J; SIEMES, C; AARNOUDSE, A; VERHAMME, K; STRICKER, B; 
STURKENBOOM, M. 2008. Adverse drug reaction-related hospitalizations: a population-based cohort study. Pharmacoepidemiol Drug Saf. 17(4):365-371.

WHO Collaborating Centre for Drug Statistics Methodology. ATC/DDD Index. Disponible desde Internet en: http:// http://www.whocc.no/atcddd/ (con acceso 02/04/08).
YEE, J; HASSON, N; SCHREIBER, D. 2005. Drug related emergency visits in an elderly veteran population. Ann Pharmacother. 39(12):1990-1995.

Recibido: Julio 11 de 2008

Aceptado: Octubre 22 de 2008 